\title{
Norois
}

Environnement, aménagement, société

$221 \mid 2011$

Innovations et agricultures urbaines durables

\section{Quelles solutions spatiales pour intégrer l'agriculture dans la ville durable? Le cas des « hameaux agricoles » dans l'Hérault.}

Which spatial solutions to integrate agriculture in sustainable town? The case of "hameaux agricoles" in the Hérault.

\section{Brigitte Nougarèdes}

\section{(2) OpenEdition \\ Journals}

\author{
Édition électronique \\ URL : https://journals.openedition.org/norois/3775 \\ DOI : $10.4000 /$ norois. 3775 \\ ISBN : 978-2-7535-1838-4 \\ ISSN : 1760-8546 \\ Éditeur \\ Presses universitaires de Rennes
}

Édition imprimée

Date de publication : 30 décembre 2011

Pagination : 53-66

ISBN : 978-2-7535-1786-8

ISSN : 0029-182X

Référence électronique

Brigitte Nougarèdes, «Quelles solutions spatiales pour intégrer l'agriculture dans la ville durable? Le cas des « hameaux agricoles » dans l'Hérault. », Norois [En ligne], 221 | 2011, mis en ligne le 30 décembre 2013, consulté le 13 janvier 2022. URL : http://journals.openedition.org/norois/3775 ; DOI https://doi.org/10.4000/norois.3775 


\title{
QUELLES SOLUTIONS SPATIALES POUR INTÉGRER L'AGRICULTURE \\ DANS LA VILLE DURABLE? \\ LE CAS DES « HAMEAUX AGRICOLES » DANS L'HÉRAULT
}

\author{
Brigitte Nougarèdes \\ UMR 0951 Innovation \\ (INRA - Institut National de la Recherche Agronomique), \\ 2 place Pierre-Viala - 34060 Montpellier Cedex 1, France \\ nougaredes@supagro.inra.fr
}

\section{RÉSUMÉ}

En France, la politique de développement urbain durable préconise la densification de l'habitat et la protection des espaces agricoles et naturels. L'application de cette politique conduit à modifier la gestion des constructions en zones agricoles et restreint les possibilités de construction de bâtiments agricoles et de logement pour les agriculteurs. Parallèlement, la profession agricole rappelle les besoins de construction de bâtiments liés à l'activité agricole qui entretient et valorise ces espaces. Dans l'Hérault, pour répondre simultanément aux enjeux de préservation du sol et de maintien de l'activité agricole, des solutions innovantes de regroupement des constructions agricoles appelées " hameaux agricoles » se développent. Ces innovations territoriales sont développées dans des espaces périurbains caractérisés par des relations sociales problématiques : lien social faible, conflits d'usage et de voisinage, etc. Ces modalités innovantes de gestion socio-spatiales des constructions agricoles renouvellent sensiblement les sociabilités locales, générant des phénomènes de solidaritélexclusion entre agriculteurs, de modification/réduction des sociabilités entre agriculteurs et résidents, de développement de l'entre-soi, et font apparaître des problématiques inattendues d'iniquité sociale. Ces phénomènes sociaux soulèvent des interrogations sur la vivabilité de ces modèles socio-spatiaux de gestion du bâti agricole. Plus globalement c'est la question de l'intégration spatiale de l'habitat agricole dans la ville durable à la française (densifiée) et des fonctions assignées à l'agriculture qui sont soulevées.

MOTS CLÉ : ville durable - périurbain - habiter - sociabilité - bâti agricole - action publique

\section{ABSTRACT}

Which spatial solutions to integrate agriculture in sustainable town? The case of "hameaux agricoles" in the Hérault

In France, the sustainable urban planning recommends the control of urban sprawl by the densification of dwellings and the protection of natural and farmed areas. The implementation of this policy leads to the modification of the managing of constructions in agricultural areas. It reduces the possibilities of building farm buildings and farmers' dwellings. At the same time, the agricultural profession insists upon the need of new buildings used for agricultural activities that maintain and valorize these spaces. In the Hérault, innovative solutions of grouping buildings together called "hameaux agricoles" are developed. These 
territorial innovations are developped in periurban areas characterised by problematic social relations: a week social link, land use and neighbourhood conflicts, etc. These innovative socio-spatial solutions modify local social relations, producing phenomena of solidarity/exclusion between farmers, of modification/reduction of social relations between farmers and inhabitants, development of "between ourselves" behaviour, and unexpected problems of social inequity. These phenomena question the livability of these socio-spatial models. More widely they question the spatial integration of farms in the French sustainable town and the functions assigned to agriculture.

KEY WORDS : sustainable town - periurban - to inhabitate - social relations - farm building - public action.

Le développement durable est au-devant des scènes scientifiques, politiques et médiatiques depuis les années 1980. Au-delà de la prise de conscience mondiale de la nécessité de maîtriser notre développement, c'est la difficulté à s'accorder sur la définition et la mise en œuvre concrète du développement durable qui alimente les nombreux débats scientifiques et politiques depuis cette période. Dès les années $1950^{1}$, des travaux ont été développés sur les enjeux environnementaux donnant naissance au concept d'éco-développement (Sachs, 1974), mais la première référence publique au concept de développement durable est issue des travaux réalisés dans le cadre de la publication de la Stratégie Mondiale de la Conservation (SMC) en 1980. Toutefois, c'est la définition issue du rapport Brundtland de la Commission Mondiale sur l'Environnement et le Développement (CMED) en $1987^{2}$ qui s'est largement imposée. Selon ce rapport le développement durable repose sur trois piliers : économique, environnemental et social. Même si les accords politiques internationaux du sommet de Rio (1992) témoignent d'un consensus sur cette conception tridimensionnelle de la durabilité, c'est dans la conception et la mise en œuvre concrète de politiques de développement durable que se manifeste la complexité inhérente à la prise en compte de ces trois dimensions.

L'application à l'aménagement territorial et urbain en est un exemple particulièrement instructif car il interroge les dimensions spatiales du développement durable. En effet, en vertu du principe d'utilisation économe des ressources naturelles non renouvelables telles que le sol, les politiques d'aménagement territorial et urbain durable font de la maîtrise de l'étalement urbain et de la périurbanisation, très consommateurs d'espace, un enjeu de premier ordre. Elles préconisent en conséquence la densification de l'habitat pour absorber la croissance démographique. Cette politique révèle non seulement des effets de concurrence entre les différents enjeux économiques, environnementaux et sociaux mais fait apparaître une dimension souvent éludée du développement durable qu'est la « vivabilité » des nouveaux modes d'organisation spatiale des activités développés dans le cadre de la densification de l'habitat.

La gestion du bâti agricole constitue un cas éclairant de cet enjeu ignoré des politiques d'aménagement durable qui participe pourtant à la durabilité des exploitations agricoles (Landais, 1998) et de la qualité de vie des quartiers (Outrequin, Charlot-Valdieu, 2006). C'est ce que cet article se propose d'illustrer en exposant les effets de nouveaux modes de gestion socio-spatiale du bâti agricole issus de la politique d'aménagement urbain durable en France. En effet, cette politique, mise en place par l'État français via la promulgation de la Loi Solidarité et Renouvellement Urbains (loi SRU, 2000), préserve indirectement les espaces agricoles et naturels par la densification de

1. En 1951, l'Union Internationale pour la Conservation de la Nature (UICN) publie le premier rapport sur l'état de l'environnement dans le Monde.

2. Le rapport Brundtland définit ainsi le développement durable : «Un développement qui répond aux besoins du présent sans compromettre la capacité des générations futures de répondre aux leurs. » 
l'habitat et directement par une restriction des possibilités de construction dans les zones agricoles. Ces zones sont inconstructibles, selon le code de l'urbanisme, seules des constructions liées à des aménagements publics ou à l'exploitation agricole y sont autorisées sous certaines conditions. Ces mesures de restriction des autorisations de construction en zone agricole touchent en premier lieu les exploitations agricoles. Or, se sont ces mêmes exploitations qui entretiennent les espaces agricoles à protéger et qui revendiquent la nécessité de construire de nouveaux bâtiments pour se développer.

Pour répondre à ces enjeux concurrentiels le département de l'Hérault, département viticole du Sud de la France, promeut des solutions innovantes de gestion du bâti agricole par le regroupement des constructions agricoles en périphérie et en continuité des espaces résidentiels. Les premiers regroupements appelés « hameaux agricoles » soulèvent de nombreuses questions : à la fois sur l'efficacité de ces solutions pour protéger les espaces agricoles et sur les conséquences de ces modes de gestion socio-spatiale sur la cohabitation entre agriculteurs et habitants. Le cas du bâti agricole renvoie de fait à la problématique plus générale de gestion socio-spatiale des activités dans la ville territoire (Chalas, 1997).

\section{Prise en compte de la sociabilité dans la conception de la politique d'aménagement territorial durable}

\section{UNE SOCIABILITÉ IMPENSÉE À L'ÉCHELLE NATIONALE...}

En 1992, lors du sommet de la terre de Rio organisé par les Nations Unies, un engagement international est scellé pour promouvoir les croissances et compétitivités nationales au service du développement durable. Cet engagement est traduit dans l'Agenda 21 et, en Europe, dans la Stratégie Européenne de Développement Durable (SEDD) ${ }^{3}$ qui se décline en Stratégies Nationales de Développement Durable (SNDD). S’appuyant sur le rapport «Équilibres ${ }^{4}$ », l'État français définit des axes prioritaires en mettant l'accent sur deux enjeux majeurs : la lutte contre le réchauffement climatique et l'aménagement durable du territoire. Dans le volet territorial de la SNDD ${ }^{5}$, la maîtrise de l'étalement urbain est définie comme « un enjeu de premier ordre pour la prévention de la ségrégation sociale et des ressources naturelles, notamment du sol, etc. » (extraits SNDD, actualisée 2006). Pour répondre à cet objectif deux principes majeurs guident l'action de l'État : densifier l'habitat et protéger les espaces agricoles et naturels. Après quarante ans d'étalement urbain, l'application des principes de développement territorial durable remet donc en question le phénomène généralisé de périurbanisation. Le volet social de la SNDD pose comme « conditions premières du développement durable, la dignité humaine, la cohésion sociale et la citoyenneté » (extrait SNDD, juin 2003). La dimension sociale apparaît aussi de manière transversale dans différents domaines d'action à travers l'objectif « d'inclusion sociale » qui recouvre : la lutte contre la pauvreté et l'exclusion, les discriminations et l'accès aux ressources : éducation, emploi, logement. Dans l'axe « aménagement du territoire », la lutte contre l'étalement urbain et la périurbanisation est prioritaire et s'appuie non seulement sur la diminution de consommation des espaces non construits, mais aussi sur le développement de l'attractivité de la ville. La ville durable doit être rendue « désirable » par une amélioration des conditions de vie via la diminution des déplacements quotidiens, une meilleure accessibilité des services, l'accroissement des déplacements piétons ou non motorisés dans l'urbain. L'amélioration de la qualité de vie, dans le volet territorial, cherche donc d'abord à répondre non pas à des enjeux sociaux mais bien à des enjeux environnementaux : rendre la ville attractive pour développer la densification urbaine.

3. La SEDD est signée en 2001 à Göteborg.

4. Equilibres : Prospective du rôle de l'État stratège face aux enjeux de développement durable, commissariat général du plan

5. La première édition de la Stratégie Nationale de Développement Durable (SNDD) est réalisée en 2003 et sera actualisée régulièrement. 
De façon globale, le volet social de la SNDD focalise donc en priorité sur des objectifs d'inclusion sociale et de qualité de vie via l'amélioration de l'accès aux ressources. On ne trouve aucune évocation directe d'enjeux relatifs à la cohésion sociale dans les fondements cette politique, même si les objectifs cités témoignent implicitement d'un soucis de cohésion sociale. Mais, il semble donc que ce qui fait société soit pensé plus autour de la satisfaction individuelle que de ce qui constitue un collectif, une communauté. Ainsi la question de la cohésion sociale est implicitement abordée par la satisfaction des ressources nécessaires à la qualité de vie, ce qui renvoie de fait à une approche par la satisfaction des besoins individuels. On est loin des idéologies ayant cours dans les années 1960 où la quête de cohésion sociale était pensée non par la satisfaction de l'individu mais par la constitution de liens via le développement de la sociabilité entre classes sociales, une sociabilité assurée par une organisation spatiale combinant proximité physique et mixité sociale dans les grands ensembles. Bien que ces modalités de traitement de la cohésion sociale aient montrées leurs limites (Chamboredon, Lemaire, 1970), il reste aujourd'hui, que la question des conséquences de la densification de l'habitat sur la sociabilité semble totalement éludée, alors que son traitement est un enjeu non négligeable pour la réussite de ce nouveau modèle de développement d'habitat moins consommateur d'espace.

\section{... MAIS SOURCE DE NOUVEAUX MOdèles LOCAUX D'HABITAT : « LES HAMEAUX AGRICOLES »}

L'application de la SNDD est mise en œuvre par la loi Solidarité et Renouvellement Urbains ${ }^{6}$ (SRU) promulguée en 2000. L'injonction de protection des espaces agricoles remet en cause les règles autorisant les constructions en zone agricole. En effet, la loi SRU introduit de nouveaux critères pour délivrer les permis de construire des bâtiments liés à l'activité agricole : l'autorisation de construire est alors soumise à l'obligation de démontrer la nécessité fonctionnelle de la construction pour l'exploitation agricole. Ce critère « qualitatif » est soumis à interprétation. Les premières interprétations de la loi varient selon les départements mais de manière générale, elles tendent à réduire surtout les possibilités de construction de logements pour les agriculteurs et leurs salariés ou dans le cadre d'hébergements à la ferme. Ceci contraint non seulement l'installation de nouvelles exploitations mais réduit aussi leurs capacités d'adaptation par la diversification des activités et des modes de commercialisation (agritourisme). Ces premières interprétations par les services de l'État en charge de faire appliquer la loi au niveau départemental (DDE, DDAF) ${ }^{7}$, font apparaître des conflits entre la profession agricole et ces services. Pour gérer ces conflits et permettre l'élaboration de solutions adaptées aux contextes locaux, une circulaire du ministère de l'Agriculture $^{8}$, s'inspirant des expériences issues d'initiatives locales, met en place des groupes de concertation inter-institutionnels départementaux : les Groupes de Travail Agriculture Urbanisme et Territoire (GTAUT $)^{9}$. La majorité des départements répond à cette nouvelle problématique de gestion du bâti agricole par un recadrage juridique qui consiste à 1) élaborer une définition précise de l'exploitation agricole, 2) traduire les critères de « lié et nécessaire » au fonctionnement de l'exploitation agricole, en fonction du contexte local. Certains départements ne se limitent pas à interpréter la loi et engagent une réflexion sur la gestion spatiale du bâti agricole.

6. Loi n² 2000-1208 du 13 décembre 2000 .

7. Dans le cadre de la réforme de l'administration territoriale de l'État (décret n 2009-1484 du 3 décembre 2009), la DDE (Direction Départementale de l'Equipement) et la DDAF (Direction Départementale de l'Agriculture et de la Forêt) ont depuis fusionné au sein de la Direction Départementale des Territoires et de la Mer (DDTM).

8. Circulaire «DGFAR/SDER/C2008-5006 » de la Direction Générale de la Forêt et des Affaires Rurales, Sous-Direction de l'environnement et de la ruralité du 14 février 2008.

9. « le groupe devrait associer au minimum le milieu agricole, les élus territoriaux, et l'État, avec la possibilité de l'élargir aux autres composantes de la ruralité. Il doit donc comporter au minimum des représentants départementaux : des services de l'État principalement concernés : DDAF et DDE (DDEA dans les départements concernés), du Président du Conseil général, de la profession agricole, des compagnies consulaires, en particulier la Chambre d'agriculture, et la SAFER, des communes et de leurs groupements (association des maires), des associations représentatives des enjeux de gestion des espaces naturels et ruraux (dont les associations environnementalistes) » (extrait de la circulaire DGFAR/SDER/C20085006, du 14 février 2008, op. cit.). 
Parmi ces départements, l'Hérault propose non seulement un nouveau modèle de gestion sociospatiale du bâti agricole appelé « hameau agricole » mais aussi un dispositif d'appui pour sa mise en oeuvre. En effet, le Groupe de Travail Urbanisme et Agriculture (GTUA) qui associe trois collèges ${ }^{10}$ propose un «paquet technique » comprenant : 1) le regroupement des constructions agricoles pouvant intégrer le logement des agriculteurs dans une zone dédiée de la commune en continuité du bâti existant, 2) un portage public des projets par la commune ou l'intercommunalité, 3) un appui financier du Conseil Général. Le portage public des projets a pour objectif, à travers la maîtrise foncière et réglementaire des regroupements, d'une part, de permettre la cession de parcelles aménagées aux agriculteurs à des prix intermédiaires entre le prix du foncier constructible et le prix du foncier agricole et, d'autre part, de garantir la destination agricole des projets à travers l'offre de trois formules juridiques ${ }^{11}$ plus ou moins contraignantes réglementant la destination agricole des bâtiments à moyen ou long terme. Cette solution est issue des problématiques spécifiques de ce département. En effet, l'Hérault est caractérisé par la croissance démographique la plus forte $^{12}$ de la région Languedoc-Roussillon qui se distingue elle-même par sa position de tête au classement des régions de France métropolitaine avec un taux de variation annuel moyen de 1,4\% pour la période de 1999 à 2007 (Source : INSEE, recensement 2007). L'étalement urbain résultant de cette forte croissance démographique se réalise essentiellement sur les espaces agricoles des communes périurbaines (Abrantes et al., 2010). Dans le département, la viticulture, production dominante, est majoritairement le fait de petites exploitations de coopérateurs traditionnellement implantées dans les centres des villages. Malgré cette configuration, l'étalement urbain s'accompagne d'un mitage des espaces agricoles. Ce phénomène est partiellement lié à la gestion des constructions agricoles. À la multiplication de constructions illicites s'ajoute le développement d'habitations lié au changement de destination de bâtiments agricoles (faux projets agricoles, faillite d'exploitations). De même, les constructions agricoles migrent vers les espaces périphériques du fait de l'évolution des besoins qualitatifs (mise aux normes environnementales) et quantitatifs (évolution du matériel agricole) en termes de bâtiments d'exploitation (Soulard et al., 2007). De plus, avec la croissance démographique et l'arrivée de nouveaux habitants, la localisation intra-urbaine des exploitations rend la cohabitation entre agriculteurs et habitants difficile, et génère des conflits de voisinage. Ceci constitue un argument supplémentaire pour sortir les exploitations des centres bourgs. Par ailleurs, le marché immobilier constamment en croissance ${ }^{13}$ incite les agriculteurs ne pouvant prétendre à la construction de leur logement en zone agricole à revendiquer leur besoin de logement au sein du GTUA via les syndicats. Le regroupement en hameaux agricoles constitue donc une solution socio-spatiale pour freiner le mitage des espaces agricoles, répondre aux besoins de bâtiments des agriculteurs et réduire les conflits de voisinages.

Dans ce contexte, on s'interroge sur la manière dont ces regroupements sont vécus par les habitants et les agriculteurs. Vont-ils permettre de solutionner les problématiques auxquelles ils sont censés répondre?

\section{Répercussions sociales des hameaux agricoles}

\section{Une Étude de CAS dans deux Communes de l'Hérault}

Les analyses qui suivent sont tirées d'une analyse comparative des sociabilités locales en bâti agricole traditionnel et en bâti agricole regroupé en hameau réalisée dans deux communes de la

10. Les trois collèges constitutifs du GTUA sont : l'État (DDTM), les élus locaux (Conseil Général et Association des maires) et la profession agricole (Chambre d'Agriculture et syndicats agricoles majoritaires : FDSEA, CDJA).

11. Le Conseil Général a financé une étude juridique pour l'élaboration de trois formules juridiques de cession des parcelles aménagées aux agriculteurs et de contrôle de la destination agricole des constructions.

12. Son taux de croissance démographique entre 1999 et 2007 est de $+1.6 \%$ par an.

13. Entre 2000 et 2006, le prix moyen des transactions pour les terrains à bâtir est passé de 51 à 122 euros le m2 en Languedoc-Roussillon. Depuis cette période, le prix moyen du $m 2$ de terrain à bâtir maintien la région au $2^{e}$ ou $3^{e}$ rang des 22 régions métropolitaines (source : observatoire statistique, logement-construction, DREAL-LR), 
Communauté d'Agglomération de Montpellier (fig. 1). Les sociabilités en bâti viticole regroupé ont été étudiées sur la commune de Saint-Géniès-des-Mourgues, commune viticole en croissance démographique située en deuxième couronne de Montpellier. L'activité viticole y est l'activité économique principale et s'organise au sein d'une coopérative viticole. La plupart des bâtiments agricoles ont disparu du centre du village grâce au premier regroupement de dix hangars agricoles (photo 1) dans les années 1990 mais quelques exploitations restent enclavées dans des lotissements. Au moment de l'étude la commune réalisait un deuxième hameau agricole (fig. 2 planche I). Il consiste en un lotissement de 12 parcelles de $1500 \mathrm{~m}^{2}$ en zone agricole. Chaque parcelle peut recevoir un hangar agricole, le logement de l'agriculteur et un gîte rural ${ }^{14}$. Les parcelles sont vendues aux agriculteurs à un prix 5 fois inférieur au prix du terrain à bâtir de parcelles établies sur la même zone à la même période. La destination agricole de ces constructions est assurée pour une durée de 12 ans via le cahier des charges du lotissement. C'est dans la commune de Saint-Georges-d'Orques située dans la $1^{\text {re }}$ couronne de Montpellier que nous avons analysé les sociabilités en bâti agricole traditionnel. Cette commune présentait en effet une évolution démographique croissante accompagnée d'un solde migratoire annuel positif, témoignant de l'apport de populations nouvelles, la présence d'une activité viticole relativement importante avec une implantation des exploitations dans tous les types d'espace : résidentiels (centre médiéval,

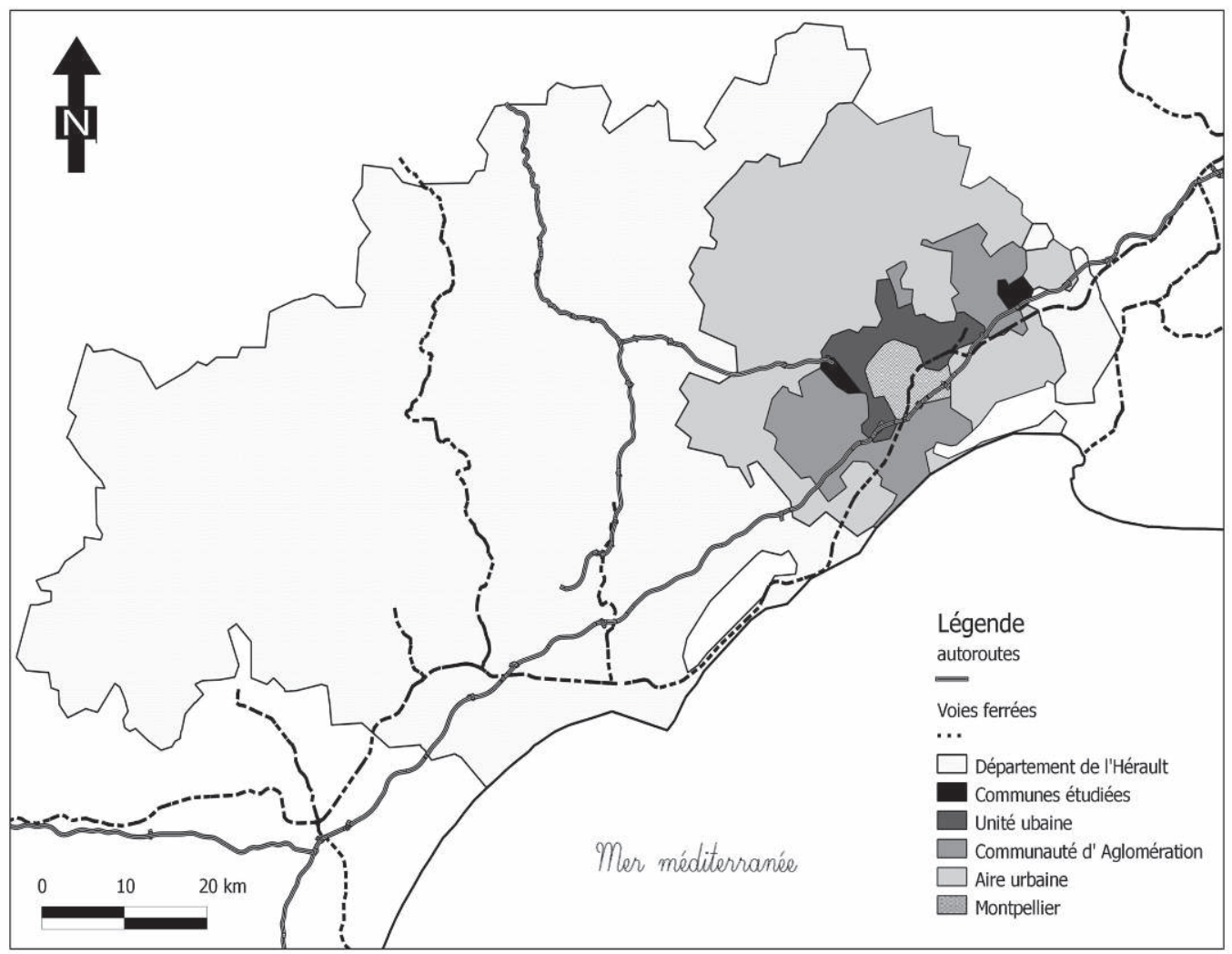

Figure 1 : Localisation des communes étudiées dans la Communauté d'agglomération de Montpellier Location map of studied communities in the urban agglomeration of Montpellier 
extension du XIX ${ }^{\mathrm{e}}$ siècle, pavillonnaire) et agricoles (mas isolés). De plus, elle avait un projet de hameau agricole au moment de l'étude.

L'étude a été réalisée à partir d'entretiens compréhensifs conduits auprès d'agriculteurs, de résidents mitoyens d'exploitations agricoles et d'élus locaux. Ils ont permis d'explorer les pratiques spatiales (pratiques agricoles ${ }^{15}$, trajectoires résidentielles, choix du lieu de vie, modes d'habiter) et sociales (relations de sociabilité) des habitants et leurs points de vue sur les projets de hameaux agricoles. Les relations de sociabilité, la perception de l'activité agricole et des projets de hameaux agricoles ont été analysés au regard des modes d'habiter. Une soixantaine d'entretiens compréhensifs ont été complétés par la recension et la cartographie de données complémentaires : cartographie des logements, des bâtiments d'exploitation, et des circulations d'engins agricoles avant et après le regroupement des exploitations lorsqu'il a eu lieu (fig. 3).

\section{LE REGROUPEMENT DE HANGARS AGRICOLES :}

\section{DES AMÉLIORATIONS LOGISTIQUES ACQUISES AU PRIX DE RELATIONS SOCIALES ALTÉRÉES}

Le premier regroupement de hangars à Saint-Géniès-des-Mourgues (photos 1 et 2) apporte indéniablement des améliorations logistiques et de conditions de travail aux agriculteurs : amélioration de la circulation et accès à des bâtiments d'exploitations adaptés et répondant aux normes en vigueur. Selon les bénéficiaires, la dissociation des bâtiments d'exploitation du logement ne perturbe pas l'organisation du travail et le regroupement des hangars apporte au contraire un plus : il participe au développement des relations de dialogue socio-technique et de nouvelles formes de solidarité. Par contre les agriculteurs qui n’ont pas souhaité ou n’ont pas pu accéder au regroupement signalent une perte de solidarité et manifestent un sentiment d'exclusion. Par ailleurs, les agriculteurs, lorsqu'ils sont installés dans le village, modifient spontanément leurs pratiques afin de ne pas gêner leurs voisins : modification des horaires d'utilisation des engins agricoles et des tournées de traitements phytosanitaires pour réduire les nuisances sonores, olfactives et les pollutions. Ces pratiques de bon voisinage sont abandonnées à la périphérie du regroupement et les relations avec les résidents mitoyens sont évitées. Deux explications apparaissent : 1) les agri-

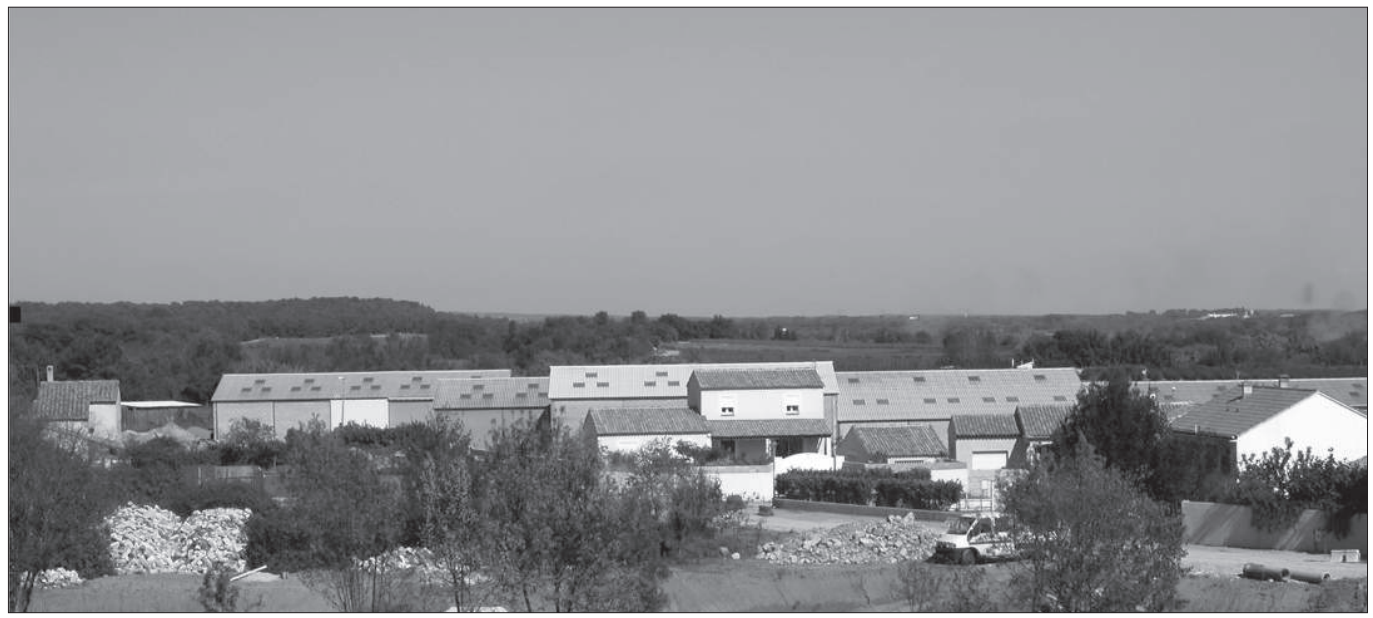

Photo 1 : Insertion du premier regroupement de hangars agricoles dans l'espace résidentiel à Saint-Géniès-desMourgues (B. Nougarèdes, 2006)

2006)

Neighborhood of the first grouping of farm buildings in at Saint-Genies-des-Mourgues (B. Nougarèdes, 


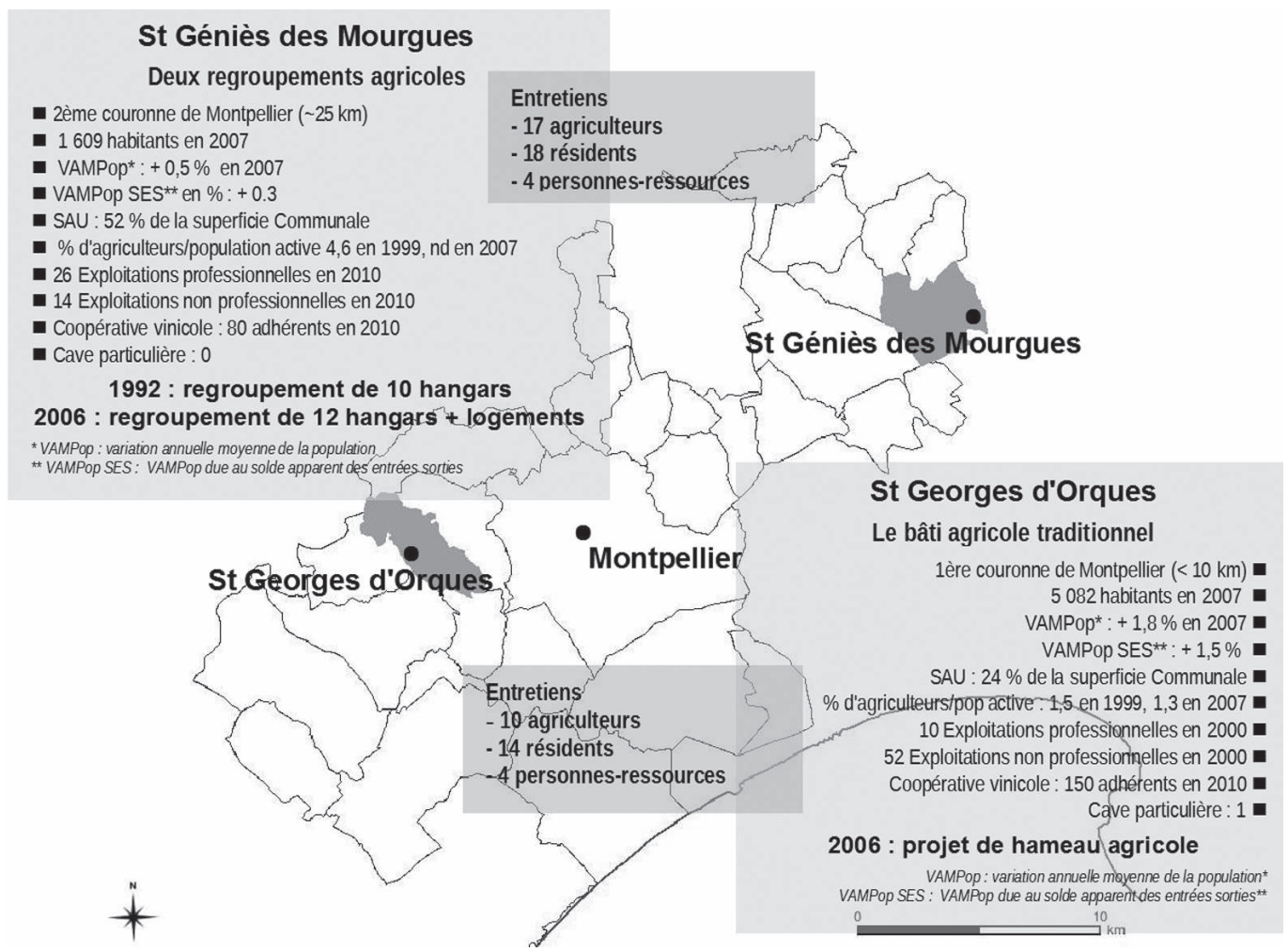

Figure 3 : Caractéristiques démographiques et agricoles des deux communes étudiées Demographic and agricultural caracteristics of the two studied communities

culteurs perçoivent ce lieu comme un espace professionnel et non comme un espace résidentiel et, de ce fait les résidents mitoyens ne sont pas considérés comme des voisins ; 2) le sentiment d'avoir été contraints à se regrouper fait naître chez certains un refus de toute autre forme de coopération ou de socialisation aux abords du regroupement : "On nous a obligé à nous installer là, maintenant qu'on ne vienne pas mennuyer si j'allume mon tracteur à 4 heures du matin. " Ceci a des conséquences sur la qualité de vie des résidents mitoyens qui subissent les effets d'une concentration des nuisances : bruit des engins agricoles démultiplié, pollutions de l'espace domestique par les produits phytosanitaires (qui peut atteindre l'intérieur de l'habitation).

Ce premier regroupement a donc amélioré les conditions de travail des viticulteurs mais crée des effets de solidarité et d'exclusion au sein des agriculteurs et un phénomène d'entre-soi au sein des bénéficiaires du regroupement. Celui-ci est fortement présent dans l'esprit de ces agriculteurs lorsqu'ils se projettent dans le deuxième hameau évoquant souvent l'aspiration à vivre entre eux : "C'est bien, on sera entre nous. »Cette réaction fait écho à un sentiment présent chez certains d'entre eux qui se sentent envahis voire dominés par les nouveaux résidents qui «veulent tout diriger » et imposer leur mode de vie. En effet, il semble, selon les enquêtes, que ces deux catégories d'habitants aient du mal à se côtoyer du fait des différences culturelles, des modes de vie et des modes d'appropriation du village. Les loisirs qu'ils affectionnent respectivement témoignent de cette divergence : aux agriculteurs, la chasse, la pétanque, les traditions taurines; aux nouveaux résidents l'informatique, le tennis, le théâtre. Ces différences culturelles réduisent les possibilités de rencontre voire les opposent. Seules quelques activités ( le football, la scolarisation des enfants) 


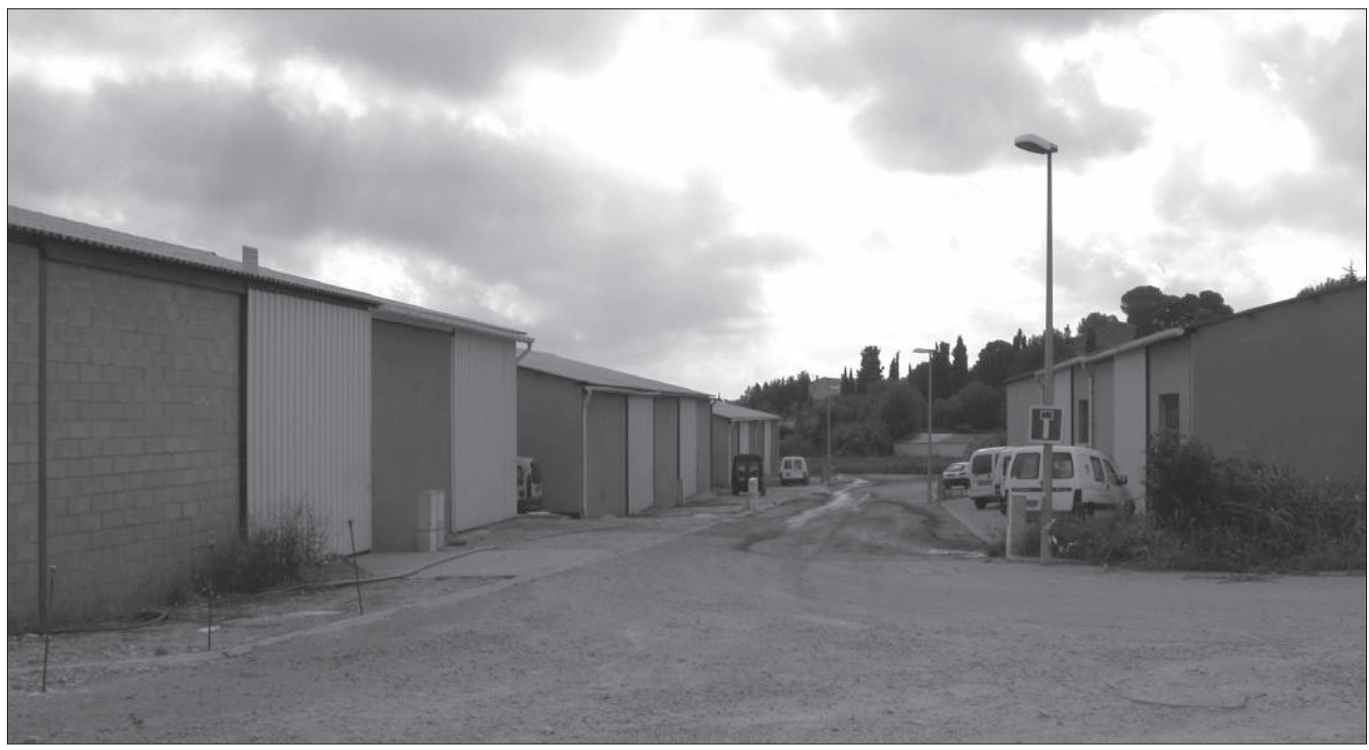

Photo 2 : Premier regroupement de dix hangars agricoles à Saint Géniès des Mourgues (B. Nougarèdes, 2006) First grouping of ten farm buildings at Saint Genies des Mourgues (B. Nougarèdes, 2006)

favorisent les échanges. La résolution des problèmes de cohabitation entre activité agricole et résidentielle par la spécialisation des espaces ne semble pas ici totalement opérationnelle. Elle se fait au prix de la constitution d'un « espace sinistré » et de l'exacerbation de dynamiques d'entre soi.

\section{LE QUARTIER INCLUANT HANGARS ET LOGEMENTS DES AGRICULTEURS : UN PROBLÉME D'ÉQUITÉ SOCIALE}

Le deuxième regroupement associant hangars et logements ne cherche pas à répondre à une contrainte en termes d'organisation du travail puisque la dissociation du logement et des bâtiments techniques n'a posé aucun problème aux agriculteurs installés dans le premier regroupement. Ce deuxième projet a donc clairement pour objectif de répondre au besoin de logement des agriculteurs en leur fournissant des parcelles constructibles à un prix inférieur au marché. Mais tous les habitants ne voient pas ce projet d'un bon œil. Les difficultés d'accès au logement concernent une grande partie de la population et touchent notamment les jeunes adultes originaires de ces villages périurbains qui ne parviennent plus à s'installer sur leur commune d'origine du fait de l'augmentation importante du prix du foncier constructible et/ou du bâti. Le problème du logement est général en France (Bigot, Hoibian, 2010) mais particulièrement problématique dans la région Languedoc-Roussillon ${ }^{16}$. Certains habitants manifestent leur désapprobation au point que le maire de la commune se trouve contraint à modifier son projet de hameau agricole. Il intègre finalement le hameau agricole à un projet d'habitat global qu'il nomme « quartier viticole » (fig. 2 - planche I). Il comprend 22 parcelles de $350 \mathrm{~m}^{2}$ « à prix maîtrisé » pour les jeunes couples de la commune à revenu modeste, 6 maisons de village en accession sociale à la propriété, 14 logements sociaux collectifs en location, et 20 parcelles au prix du marché qui permettent d'équilibrer globalement le budget. Le Maire souligne que « cette opération [le hameau agricole] ne fait pas l'unanimité dans le village [...] mais que cette critique s'estompe bien avec l'aménagement du quartier 
pour les jeunes à côté» et que "si on n'avait pas fait le côté social pour les jeunes cela aurait été encore plus dur». Le projet initial qui cherchait à répondre au besoin de logement des agriculteurs a donc soulevé un problème d'équité sociale. Les enquêtes ayant été réalisées au moment de la construction du deuxième projet de hameau agricole nous n'avons pu aborder la question de la cohabitation entre agriculteurs et résidents dans cette nouvelle organisation spatiale qui regroupe et concentre dans un périmètre restreint douze exploitations agricoles, et différents types d'habitat collectif et individuel, aidés et non aidés. L'organisation spatiale innovante de ce quartier agricole combine une proximité spatiale tout en maintenant une forte spécialisation socio-spatiale assumée par le Maire qui défend un objectif de mixité sociale. Celle-ci mixe aussi constructions résidentielles et professionnelles. Cette organisation originale tant d'un point de vue sociologique que spatial soulève de toute évidence des interrogations sur la manière dont va se dérouler la cohabitation dans ce quartier. Ce projet répond à la demande de logement au risque d'aggraver les risques de nuisances car ce quartier est localisé entre le hameau agricole et la coopérative viticole.

\section{Les projets de hameaux agricoles révélateurs des conceptions du « vivre ensemble"}

Les entretiens réalisés révèlent des formes d'appropriation de l'espace communal variées pour lesquelles la localisation et les fonctions de l'agriculture diffèrent en fonction de la manière d'habiter. Chaque mode d'habiter révèle de fait un certain rapport au lieu de vie. Il témoigne de la façon dont les individus se représentent le village, l'investissent spatialement, socialement, et en conçoivent sa gestion bref, se l'approprient. Quatre types de rapport au lieu de vie ont été mis à jour. Ils révèlent le lien étroit entre la manière d'habiter, la conception du vivre ensemble et l'intégration de l'activité agricole dans l'organisation socio-spatiale de la commune. Ces quatre types de rapport au lieu de vie font apparaître deux conceptions du «vivre ensemble » : « le village » et « la villégiature »(Candau, Nougarèdes, 2008).

\section{DEUX CONCEPTIONS DU «VIVRE ENSEMBLE » : LE VILLAGE ET LA VILLÉGIATURE}

Les habitants ayant une conception du vivre ensemble proche du concept de « village » recherchent ou cherchent à perpétuer le caractère rural de la commune et sa dimension communautaire. L'attraction pour le caractère rural intègre de fait l'activité agricole comme un élément fondamental de la ruralité. De ce fait, ces individus ont une tolérance importante aux gênes produites par l'activité agricole voire même en apprécient les bruits, les odeurs que d'autres considèrent comme dérangeants. Deux types d'habitants adhèrent à cette conception du vivre ensemble : i) les autochtones qui, du fait d'une pluri-activité viticole traditionnelle dans la région, ont tous un lien de proximité (sociale, culturelle) avec la viticulture. Pour eux l'agriculture doit être maintenue dans les villages et soutenue alors que les hameaux agricoles participent d'un processus plus global qui tend à faire « des villages pour riches » dont ils se sentent progressivement exclus. Au final, la majorité accepte ces projets et soutien de façon inconditionnelle les agriculteurs; ii) Des jeunes couples non originaires de la commune qui cherchent à s'ancrer dans un lieu de vie propice à la vie de famille et à l'éducation des enfants. Ils adhèrent aussi volontiers aux projets de hameau dans le but de soutenir l'activité agricole, mais restent vigilants sur les risques de spéculation par les agriculteurs, n'ayant pas oublié l'investissement financier qu'ils ont dû eux-mêmes consentir pour s'installer dans le village. Comme pour les autochtones, une partie d'entre eux soulève la question de l'équité d'accès au logement.

Les habitants ayant une conception du « vivre ensemble » proche du concept de la «villégiature » ne perçoivent ni ne recherchent la dimension rurale de leur lieu de vie. Deux types d'habitants adhèrent à cette conception du « vivre ensemble » : 1) Ceux pour qui la commune représente uniquement une localisation stratégique qui leur permet d'optimiser l'accès aux ressources nécessaires à leur qualité de vie (commerces, établissements scolaires, loisirs, etc.). Ces habitants ont 
un rapport au lieu de vie «fonctionnel », ils ne cherchent pas immédiatement à s'intégrer dans la commune voire refusent toute relation " pour ne rien devoir à personne ». Pour eux, le village "n'est pas un village rural, mais un village en ville», il est donc vu comme une forme de ville. De plus, le maintien de l'activité agricole dans les bourgs est perçu par certains comme un retard pris sur le progrès : "On n'est plus au XIXe siècle où les animaux erraient dans les villages. " Pour les plus radicaux, la sortie de l'activité hors du bourg aurait déjà du être opérée et ne doit donner lieu à aucune compensation; 2) D'autres habitants n'ont pas choisi une commune mais une maison leur permettant d'agrandir leur espace domestique, si possible en y intégrant un jardin privatif et une configuration spatiale permettant de s'isoler le plus possible du voisinage. Ils ont un rapport au lieu de vie fondé sur le retranchement sur la sphère privée. Pour eux la commune est réduite à un cadre paysager : «le calme, la campagne », la vie sociale de leur commune ne les intéresse pas ou peu. Dans ces deux cas de figure, certains résidents se considèrent incompétents pour juger de la pertinence des projets de hameaux agricoles et ne s'y intéressent pas. D'autres y sont favorables car ils y voient une contre-partie à la préservation et l'entretien du paysage et du cadre de vie, mais réclament la plus grande vigilance sur les risques de spéculation foncière inhérents à ces projets. Ces habitants, pour qui le lieu de vie est, soit réduit à une localisation stratégique, soit à l'espace domestique, apparaissent comme des habitants dé-territorialisés.

\section{Du BÂTI AGRICOLE À LA POLITIQUE LOCALE DE L'HABITAT}

Au final, le traitement de la demande de logement des agriculteurs conduit à modifier le projet pour répondre à la problématique générale de traitement du logement sur la commune, en évacuant la question des nuisances qui n'est non seulement pas réglée mais risque même d'être amplifiée. De plus, ce modèle ne résout pas la question de la transmission des exploitations. En effet, la demande de logement des jeunes viticulteurs est liée à la décohabitation entre cédant et repreneur et à des pratiques de transmission des biens. On constate généralement que ceux qui reprennent l'exploitation familiale héritent des terres, les autres héritiers reçoivent les biens immobiliers (Lafage, 2006). Or, ce modèle reproduit ce phénomène, car une fois à la retraite les viticulteurs installés dans le hameau agricole ne quitteront pas leur logement. On peut donc s'interroger sur le devenir de ces hameaux. On aurait pu imaginer que le logement des agriculteurs soit traité dans le même cadre que celui des autres habitants. Or, force est de constater que les viticulteurs, ont une forte capacité de lobbying. Dans le cas étudié, ils constituent la principale activité économique de la commune et participent activement à sa gestion (sur 19 conseillers municipaux, 9 sont agriculteurs au moment de l'enquête). Par ailleurs, il semble que dans la commune ce nouveau quartier viticole soit, comme dans le premier projet, le fruit d'un compromis qui satisfait une majorité de la population ${ }^{17}$ au risque de sacrifier les conditions de vie d'une minorité. En effet, on peut s'interroger sur le risque à choisir une forme d'organisation socio-spatiale qui comporte des risques liés au cumul d'une mixité fonctionnelle, et d'une mixité sociale organisée dans la cadre d'un dispositif d'accès au logement ségrégatif dont l'équité sociale est discutable.

Concernant les deux modèles socio-spatiaux étudiés, plusieurs enseignements peuvent être tirés. Le premier regroupement de hangars qui cherche à résoudre les problèmes de cohabitation en sortant l'activité agricole de l'espace résidentiel ne parvient pas totalement à son objectif du fait de sa localisation en continuité des habitations. Par contre, il facilite la transmission des exploitations et offre une solution plus durable de protection des espaces agricoles. À l'inverse le deuxième projet n'est pas fondé sur la mise à l'écart de l'activité agricole mais au contraire sur une politique qui tend à induire l'acceptation de l'activité agricole par la confrontation. Le hameau agricole pallie la perte du droit à construire des agriculteurs tout en préservant leur position sociale dans la commune et maintient un cadre de vie à la majorité de la population aux détriments d'une minorité.

17. En 2008, les élections municipales ont lieu. Une liste d'opposition se constitue et conteste notamment le projet de hameau agricole. Le maire est ré-élu à $78 \%$ des suffrages exprimés. 


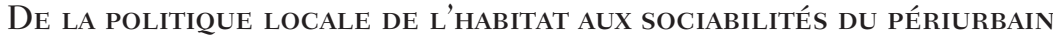

À travers les enquêtes conduites dans le cadre de cette étude, nous avons retrouvé les figures classiques d'habitants du périurbain déjà identifiées dans le cadre des travaux conduits sur les dynamiques sociales et spatiales et les modes d'habiter du périurbain. On retrouve ainsi l'attitude de retranchement sur la sphère privée (Charmes, 2005, Dodier, 2007/1) d'individus à la recherche d'un espace de repos social (Banos et al., 2009); les habitants ayant un rapport fonctionnel au lieu de vie dont la principale caractéristique est sa localisation stratégique (Jaillet, 2004); les ménages en recherche d'un ancrage local (Giusepelli, 2006/2), et enfin les autochtones dont le lieu de vie (hérité) est partie intégrante de leur identité. Ces derniers nous rappellent comme le soulignait Martine Berger (2004) que le périurbain n'est pas seulement alimenté par l'arrivée d'exurbains mais par le non-départ des ruraux. Mais les différents types de rapport au lieu de vie identifiés ici font aussi écho aux travaux réalisés sur l'appartenance locale (Sencébé, 2004) et nous éclairent sur la diversité des modes d'appropriation d'un même lieu de vie et sur leurs conséquences en terme de cohabitation et de gestion du territoire. Ces différents types de rapport au lieu de vie s'expriment dans la manière de percevoir les autres habitants, ce qui conditionne relativement l'évolution des sociabilités locales, et s'inscrivent dans les projections des habitants sur la gestion et l'aménagement du territoire. Ces projections sur la gestion et l'évolution du territoire peuvent entrer en concurrence comme le montrent d'autres travaux (Sencébé, 2004; Sotiropoulos, 2007/2) et le rapport de force peut donner lieu à un compromis local dont on peut s'interroger s'il permettra de garantir la cohésion et la paix sociale. On note que l'ancrage local n'est pas la seule ressource pour influencer la politique locale. Les habitants ayant un rapport fonctionnel au lieu de vie, les moins ancrés localement, ne sont pas pour autant les moins aptes à exercer leur pouvoir pour préserver ce qui motive leur maintien dans ce lieu : le cadre de vie entendu non pas comme un environnement relationnel mais bien comme un contexte (Forrest, 2007/1). Pour les agriculteurs fortement ancrés dans la commune, leur capacité à exercer un poids sur la gestion de la commune est variable. À Saint-Geniès-des-Mourgues, ils ont un poids considérable, car leur activité est la principale activité économique de la commune, de plus, ils détiennent le foncier et bénéficient de leur ancrage historique dans le pouvoir local. Ces jeux d'influence liés aux attentes des habitants fondées sur leur rapport au lieu de vie, se combinent à la capacité d'influence des catégories ou groupes sociaux. Ainsi les familles les plus modestes se retrouvent logées entre la coopérative et le hameau agricole. En regroupant dans un quartier la principale activité économique de la commune (et ses nuisances potentielles), et les logements attribués aux familles les plus modestes, tout en accordant des conditions privilégiées d'accès au logement aux agriculteurs, c'est tout le reste de la commune qui peut bénéficier d'un cadre de vie souhaité. En affichant une politique de mixité sociale et fonctionnelle, c'est le modèle de la villégiature et la sociabilité qui y est associée qui sont préservés.

\section{Conclusion : quelles solutions spatiales pour intégrer l'agriculture dans la ville durable?}

La protection des espaces agricoles préconisée dans le cadre du développement urbain durable fait apparaître une nouvelle problématique : la gestion socio-spatiale du bâti agricole. C'est une question vive dans les espaces périurbains dans lesquels les mesures de préservation des espaces agricoles entrent en concurrence avec les besoins de transmission et d'adaptation d'exploitations périurbaines souvent fragilisées. Les hameaux agricoles constituent une tentative de réponse à ce problème complexe. Les initiatives locales témoignent du fait que cette innovation territoriale (Jarrige et al, 2009) ne peut se limiter à la production d'un seul modèle socio-spatial et que sa conception doit être négociée au sein de dispositifs de gouvernance multi-niveaux (Chia et al., 2010). 
Les projets étudiés révèlent une convergence forte entre la politique nationale et les dynamiques locales marquées par la prédominance des fonctions paysagères, environnementales (Mathieu, 1998) et de cadre de vie des espaces agricoles. Les politiques locales entrent en synergie avec la politique nationale de développement durable car la qualité paysagère fait indéniablement partie des atouts mis en avant par les communes et les intercommunalités pour maintenir ou se prévaloir de leur attractivité. Ces objectifs environnementaux convergent avec les attentes de la majorité des habitants qui sont sensibles à leur cadre de vie et font écho au modèle de ville durable à la française fondé sur " une qualité de vie » (Emelianoff, 2003) dans l'objectif toujours croissant de satisfaire les besoins individuels. Ces conceptions du « vivre ensemble » ne risquent-t-elles pas de conforter un rapport « consumériste » au lieu de vie tel celui des habitants se référant à la villégiature? Ces modes d'organisation spatiale des activités ne risquent-elles pas d'entretenir une exigence toujours croissante de qualité de vie qui laisse peu de place à la tolérance et à la confrontation à l'autre? L'approche très fonctionnaliste de la ville durable à la française laisse peu de place à la réflexion pour d'autres modes de cohabitation alors que les pratiques développées spontanément témoignent d'une capacité des populations à gérer et inventer des solutions pour améliorer la cohabitation entre habitants et activités économiques. Pourtant, un autre pays de l'Union Européenne, la Grande Bretagne, s'appuie au contraire sur des « approches fondées sur la communauté » comme « cadre qui favorise l'expression de la sociabilité et l'apaisement des tensions, via le sentiment d'appartenance locale et les bonnes relations de voisinage, la qualité et l'éducation à l'environnement et la prise en charge des problèmes par les populations ellesmêmes », (Emelianoff, 2003). L'innovation est peut-être à trouver dans la traduction concrète de ces fondements pour une ville durable plus vivable qui favorise l'intégration de l'activité agricole et plus largement le lien social et l'ancrage local plutôt que l'individualisme et l'attitude consumériste d’habitants « dé-territorialisés ».

\section{Bibliographie}

Abrantes P. Soulard C., Jarrige F. Laurens L., 2010. Dynamiques urbaines et mutations des espaces agricoles en Languedoc-Roussillon (France), Cybergeo, Espace, Société, Territoire, article 485, mis en ligne le 13 janvier 2010. [http://www.cybergeo.eu/index22869.html].

Banos V., Candau J., Baud A.-C., 2009. Anonymat en localité, Cahiers internationaux de sociologie 2/2009 (n 127), p. 247-267.

Bigot R., Hoibian S., 2010. La crise du logement entretient le sentiment de déclassement social, Crédoc, Consommation et modes de vies, $\mathrm{n}^{\circ} 226$, février 2010.

Candau J., Nougarèdes B., 2008. La ségrégation spatiale : une solution pour la paix sociale? L'expérience des hameaux agricoles dans la périphérie de Montpellier, Colloque Les périphéries urbaines entre normes et innovations. Les villes du sud de l'Europe, Bordeaux 11-13 juin 2008, 26 p.

Chamboredon J.-C., Lemaire M., 1970. Proximité spatiale et distance sociale. Les grands ensembles et leur peuplement, Revue Française de Sociologie, XI, p. 3-33.

Chalas Y., 1997. Territoires contemporains et représentations : des vieux paradigmes urbanistiques aux nouvelles figures de la ville, revue de la géographie alpine, $\mathrm{n}^{\circ}$ 4, p. 11-36.

Charmes E., 2005. La vie périurbaine face à la menace des gated communities, Paris, L’Harmattan, coll. «Villes et Entreprises », 219 p.

Chia E., Mathe S., Rey-Valette H., Michel L., Soulard Ch., Nougaredes B., Jarrige F., Clement C., Barbe E., Martinand P., Maurel P., Guilheneuf P.-Y., 2010. Comment étudier la gouvernance territoriale? Mise à l'épreuve d'une grille de lecture, Colloque Identité, Qualité et compétitivité territoriale. Développement économique et cohésion dans les territoires alpins, joint Colloque ASRDLF - AISRe, Aoste 20-22 septembre, Session spéciale : Développement territorial et gouvernance foncière, 23 p. 
Dodier R., 2007. Quelle articulation entre identité campagnarde et identité urbaine dans les ménages «périurbains »?, Norois [En ligne], 202/1, mis en ligne le $1^{\mathrm{er}}$ mars 2009, [http://norois.revues.org/ index1616.html].

Emelianoff C., 2003, Les villes durables européennes : diversité d'approches, La ville durable en Europe, La revue Durable $\mathrm{n}^{\circ}$ 5, mai-juin, p. 14-18.

ForRest R., 2007. Le voisinage? Quelle importance?, Revue internationale des sciences sociales, 1, n 191, p. 137-151.

Giusepelli E., 2006. Place et fonctions de l'agriculture en zones périurbaines de montagne : modes d'habiter et représentations du rural, Espace géographique, 2 (Tome 35), p. 133-147.

Jaillet M.-C., 2004. L'espace périurbain : un univers pour les classes moyennes, Esprit La ville à trois vitesses : gentrification, relégation, périurbanisation, nº 303, mars-avril 2004, p. 40-62.

Jarrige F., Laurens L., Nougarèdes B., Soulard C., Sabatier B. 2008. Les projets agri-urbains : des innovations territoriales? Exemple du bâti agricole dans l'Hérault (France), dans Loudiy S., Bryant C., Launens L., (éd.) Territoires périurbains et gowvernance : perspectives de recherche, Trois-Rivières (Canada), Université de Montréal/Université du Québec, p. 83-90.

LAFAGE C., 2006. Formes et dynamiques spatiales de l'habitat agricole en contexte périurbain: cas de l'Hérault, Mémoire de Master II Recherche Études Rurales, option Géographie, 120 p.

LANDAIs E., 1998. Agriculture durable : les fondements d'un nouveau contrat social?, Courrier de l'environnement de l'INRA, n 33 , p. 5-22.

Mathieu N., 1998. La notion de rural et les rapports ville-campagne en France Les années quatre-vingt-dix, Économie rurale, $\mathrm{n}^{\circ} 247,1998$. p. 11-20.

Outrequin P., Charlot-Valdieu C., 2006. Développement durable et renouvellement urbain, des outils opérationnels pour améliorer la qualité de vie dans nos quartiers, Paris, L'Harmattan, coll. «Villes et Entreprises », $296 \mathrm{p}$.

Sachs I., 1974. Environnement et styles de développement, Annales Economie, Sociétés, Civilisations, $29^{e}$ année, $n^{\circ} 3$, p. 553-570.

Sencébé Y., 2004. Être ici, être d'ici : les formes d'appartenance sur un territoire de circulation, le Diois (Drôme), Ethnologie Française, vol. 4, n 1, 2004, p. 23-29.

Sotinopoulou E.-C., 2007/2. Espaces villageois et conflits d'appropriation : à la croisée de nouveaux désirs et de regards renouvelés, Revue Géographie Économie Société, vol. 9, p. 165-185.

Soulard C.-T., Delfosse C., Nougaredes B., Lafage C., 2007. Formes et dynamiques du bâti agricole périurbain : projets locaux d'aménagement et besoins spatiaux des agriculteurs, dans MAdELine P., MoriCEAu J.-M. (dir.), Bâtir dans les campagnes, Caen, MRSH, coll. « La bibliothèque du Pôle Rural », p. 1-15.

Cet article a été reçu le 9 février 2011 et définitivement accepté le 25 octobre 2011. 